BLOOD-BRAIN BARRIER

\section{Plugging the leak}

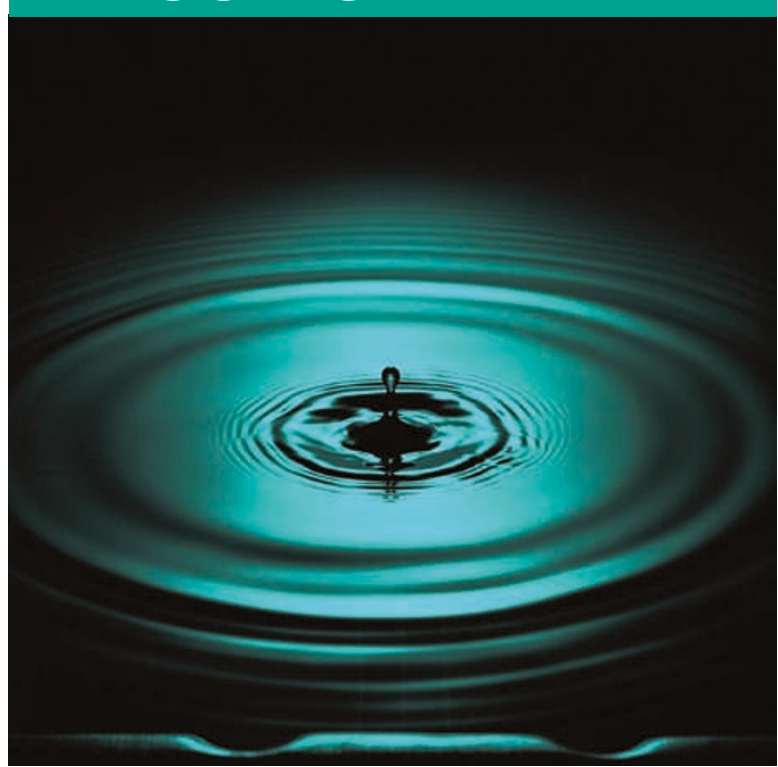

Endothelial cells in the CNS are endowed with properties - such as tight junctions, low rates of transcytosis and the expression of various transporters - that allow them to regulate the movement of molecules, ions and cells between the blood and the brain, thus contributing to the blood-brain barrier (BBB). Pericytes, which surround capillaries, are also a component of the BBB, but their role in barrier function has remained unclear. Two papers published in Nature now show that pericytes are necessary for both the formation and regulation of the $\mathrm{BBB}$.

Daneman et al. showed first that in the rat and mouse cerebral cortex, endothelial cells express tightjunction molecules and the BBB- specific marker glucose transporter 1 (Glut1) as early as embryonic day 11 . The low-molecular-mass tracer biotin, which is excluded from the CNS parenchyma by the BBB in postnatal animals, was also excluded from the brain in embryos as early as embryonic day 15 . These data show that, contrary to the prevailing hypothesis that astrocytes induce the formation of the BBB postnatally, a functional $\mathrm{BBB}$ is present during embryogenesis, before astrocytes are generated.

The authors next set out to examine whether pericytes could be involved in the prenatal regulation of BBB function and structure. They studied $P d g f r b^{-1-}$ mice, which lack CNS pericytes because beta-type platelet-derived growth factor receptor (PDGFR $\beta$ ) signalling is essential for pericyte recruitment to developing vessels. These mice died at birth, and examination of the embryos showed that they had increased vascular permeability to biotin. In neonatal Pdgfrb hypomorph mice, the number of pericytes was inversely correlated with the permeability of the BBB, suggesting that pericytes are necessary for the proper formation of the BBB. The findings of the second paper, by Armulik et al., support the notion that pericytes are important in the regulation of the BBB. This group used adult, viable pericyte-deficient mice that had mutations in the PDGFB-PDGFR $\beta$ pathway, to show that pericyte deficiency increases the permeability of the BBB to water and to a range of tracers of different molecular masses.

Both research groups also investigated how an absence of pericytes might cause this leakiness. Although most BBB-specific markers were unaffected by a lack of pericytes, they found alteration in the expression of some genes, such as those encoding proteins that regulate vascular permeability and CNS immune cell infiltration. Moreover, Daneman et al. observed that $P d g f r b^{-/-}$mice had abnormalities in endothelial tightjunction formation and increased expression of plasmalemma vesicle-associated protein, which is necessary for vesicular trafficking in CNS endothelial cells. Armulik et al. showed that the increased leakiness in their mouse models was due to increased endothelial transcytosis, and this process was inhibited by treating the mice with the tyrosine kinase inhibitor imatinib. They also found that the expression of several astrocyte markers were downregulated in pericyte-deficient mouse brains. Three of these markers are known to regulate the polarization of astrocyte end-feet - cytoplasmic processes that attach to CNS blood vessels - suggesting that pericytes might mediate the attachment of astrocyte end-feet to blood vessels.

Together, these studies indicate that the functional integrity of the $\mathrm{BBB}$ is regulated by pericytes during development and in adulthood. This might have implications for understanding and treating various neurological disorders - such as stroke, brain tumours, multiple sclerosis and diabetic retinopathy - in which breakdown of the $\mathrm{BBB}$ is a common component.

Ezzie Hutchinson

ORIGINAL RESEARCH PAPERS Daneman, R. $e$ al. Pericytes are required for blood-brain barrier integrity during embryoogenesis. Nature 13 Oct 2010 (doi 10.1038/nature09513)|Armulik, A. et al. Pericytes regulate the blood-brain barrier. Nature 13 Oct 2010 (doi 10.1038/nature09522) 